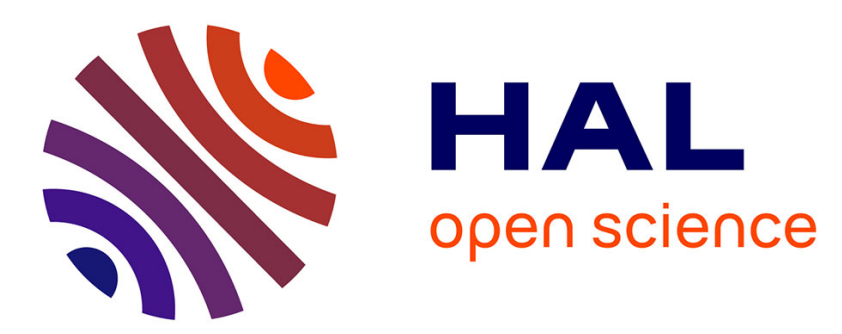

\title{
Ascension et déclin d'un notable sportif investi dans l'arène de la voile brestoise. De la fin des années 1950 au début des années 1970
}

Claude Lafabrègue

\section{- To cite this version:}

Claude Lafabrègue. Ascension et déclin d'un notable sportif investi dans l'arène de la voile brestoise. De la fin des années 1950 au début des années 1970. Sciences sociales et sport, 2021, №18, pp.149 175. 10.3917/rsss.018.0149 . hal-03343084v1

\section{HAL Id: hal-03343084 \\ https://hal.science/hal-03343084v1}

Submitted on 13 Sep 2021 (v1), last revised 15 Sep 2021 (v2)

HAL is a multi-disciplinary open access archive for the deposit and dissemination of scientific research documents, whether they are published or not. The documents may come from teaching and research institutions in France or abroad, or from public or private research centers.
L'archive ouverte pluridisciplinaire HAL, est destinée au dépôt et à la diffusion de documents scientifiques de niveau recherche, publiés ou non, émanant des établissements d'enseignement et de recherche français ou étrangers, des laboratoires publics ou privés. 
ASCENSION ET DECLIN D’UN NOTABLE SPORTIF INVESTI DANS L'ARENE DE LA VOILE BRESTOISE.DE LA FIN DES ANNEES 1950 AU DEBUT DES ANNEES 1970.

\section{Introduction}

Dans la littérature spécialisée, il est encore fréquent de caractériser le pouvoir du notable par son insertion dans le monde de la représentation politique, comme si l'un n'allait pas sans l'autre (Halévy, 1930 ; Tudesq, 1993 ; Phélippeau, 2002 ; Briquet, 2012 ; Bensoussan, 2015 ; Mattina, 2016). L'extrême focalisation sur cette fonction n'est pas sans inconvénient : elle conduit à traiter les fonctions électives exercées en dehors de la sphère politique, dans des comités d'intérêt de quartier (Mattina, 2004) ou de grands clubs sportifs (Augustin et Garrigou,1985 ; Reneaud, 1995) par exemple, comme un simple tremplin devant accélérer l'accès de ces dirigeants associatifs au statut d'édile local, faisant d'eux des notables accomplis.

Pourtant, la littérature consacrée aux élites sociales locales fait état de quelques exceptions. Certains travaux attirent l'attention sur l'assise économique des acteurs sociaux, qui constitue une condition essentielle pour acquérir de l'influence, de la notoriété et du prestige. Dans le papier qu'il consacre aux dirigeants des clubs sportifs bordelais à la fin du XIX ${ }^{\mathrm{e}}$ siècle, près de 70 ans avant que s'ouvre la période qui nous intéresse, Jean-Paul Callède (1995) montre que deux types d'élites économiques sont placées à leur tête : d'une part, la haute bourgeoisie de vieille souche qui trouve dans cette position sportive éminente une confirmation de sa notabilitéet, d'autre part, des Bordelais de fraîche date, récemment intégrés à la bourgeoisie libérale, pour qui l'accès à une place de dirigeant sportif constitue le moyen de conquérir un tel statut. Dans leur typologie des clubs de rugby du début des années 1980, Jean-Pierre Augustin et Alain Garrigou (1985) retrouvent cette liaison étroite entre pouvoir économique, mandature sportiveet notabilité. Manuel Schotté (2016) fait une observation semblable auprès des petits patrons dont l'arrivée à la tête des clubs de football professionnels consacre une trajectoire de notabilisation antérieure. Le fait de se situer au confluent de multiples intérêts économiques et politiques en prenant la direction d'un club de ce genre rend plus visible cette position de notable sans qu'ils aient besoin de briguer une fonction élective dans la sphère politique, même si certains d'entre eux s'y essaient ultérieurement. Pierre Grémion (1976) va dans le même sens quand il range dans la liste des 
notables départementaux quelques acteurs influents sur le plan économique mais dépourvus de mandat politique, comme le fait aussi Michel Robert (1970) pour les directeurs de coopératives agricoles. Toutefois, il réserve le plus souvent cette qualité à un petit nombre de personnes présentes dans les institutions de représentation élective. Mais, à la différence des premiers auteurs cités, il ne fait pas de la représentativité politique l'unique voie d'accès à la notabilité. Font aussi partie des notables ceux qui ont été élus pour représenter un secteur de la société auprès des autorités politiques (chambre d'agriculture, chambre de commerce et de l'industrie). C'est aussi ce que relève Jean-Paul Callède (2009) à propos des présidents de grands clubs sportifs universitaires, mais sans qu'on puisse leur attribuer un quelconque pouvoir économique lié à leur position professionnelle.

Effectuée à partir de sources multiples (voir l'encart 1), l'étude du cas de Louis Sparfel, un dirigeant sportif ayant exercé des fonctions électives pendant près de 17 ans, nous servira à réfléchir à la possibilité d'accéder au statut de notable local sous la $\mathrm{V}^{\mathrm{e}}$ République débutante, tout en étant privé des positions politique et économique qui sont censées procurer aux élites sociales une influence sur les échanges de tous ordres qui se nouent au sein de l'espace local. Le pouvoir que retirent les dirigeants sportifs des usages qu'ils font de leur position de représentant d'un secteur de la société locale est donc placé au centre de l'analyse. Il s'agit de déceler ce qui dans ces usages relève des pratiques typiques de la notabilité. Inversement, il convient de cerner les limites auxquelles se heurte l'exercice du pouvoir à l'échelle locale à partir d'une position élective excentrée par rapport aux autorités politiques.

La notabilité sportive est envisagée comme le produit d'une activité qui tire son efficacité des contacts récurrents établis par un dirigeant associatif avec les instances officielles qui contrôlent la distribution des avantages dont dépendent les clubs sportifs et qui lui procurent un surcroît de légitimité en satisfaisant ses demandes. Avant de juger de cette efficacité dans le cas du dirigeant examiné ici, nous décrivons ses propriétés politiques, économiques et associatives.Puis, nous présentons les éléments qui concourent à lui donner une position de notable local. Nous terminons par l'examen des circonstances qui contribuent à sa perte d'influence. 


\section{Encart 1 : L'enquête et sa méthode}

Sur le plan empirique, les moyens déployés pour décrire les rapports de pouvoir entre les divers acteurs intéressés au développement de la voile de plaisance à Brest combinent quatre sources d'information. Les trois premières ont été utilisées de manière interactive. Les archives et la presse locale ont permis d'orienter la conduite des entretiens sur des points particuliers qu'il nous paraissait intéressant d'approfondir à partir d'une connaissance préalable des faits, et de mettre aussi à distance les propos des interviewés. Réciproquement, les entretiens ont eu comme principale vertu d'attirer notre attention sur certains événements jugés éclairants, au sujet desquels nous avons cherché à glaner des informations complémentaires dans la presse et dans les archives. En outre, ils nous ont permis de mener l'enquête sur certains aspects de la réalité qui seraient restés inaccessibles si l'on s'était contenté d'examiner les textes disponibles.

Des entretiens ont été menés avec 12 acteurs qui ont tous été en contact, de près ou de loin, avec Louis Sparfel quand il était associé à la direction de la Société des régates de Brest (SRB) ou du Centre de formation nautique brestois (CFNB). Il s'agit de deux anciens présidents de la SRB, d'un salarié permanent de la SRB nommé directeur du centre nautique municipal à sa création, d'un ancien élève du CFNB passé moniteur bénévole à la $\mathrm{SRB}$, de deux anciens maîtres d'éducation physique dépendant du service départemental de la jeunesse et des sports (SEJS), chargés d'animer des séances d'initiation à la voile, de quatre anciens membres du comité directeur du club de dériveurs issu de la scission avec la SRB, et de deux professeurs d'EPS détachés à plein temps au centre nautique scolaire annexé à un lycée de la commune.

Tirés du Télégramme, les articles de presse traitant de la pratique de la voile dans la commune entre 1947 et 1975 ont donné lieu à un dépouillement quasi systématique qui nous a renseigné sur l'activité des clubs, la composition de leur direction, leur palmarès, leurs problèmes et leurs projets, leurs relations avec les autorités politico-administratives, et les discours prononcés à l'occasion des diverses cérémonies auxquelles donne lieu la vie d'un club.On a également procédé à un examen minutieux des documents déposés aux Archives municipales (AM), relatifs aux clubs de voile, à l'Office municipal des sports (OMS) et aux élections locales. Ce travail a permis, notamment, d'objectiver les relations qui se sont établies au fil du temps entre les clubs de voile et la municipalité, et d'observer les réactions 
des uns et des autres aux décisions ou aux sollicitations de l'autre partie.

Une fois que ce travail a mis à jour la place occupée par Louis Sparfel dans l'arène de la voile brestoise, une nouvelle recherche a été entreprise afin de mettre en évidence ses principales propriétés sociales. En l'absence de renseignements le concernant dans les notices bibliographiques disponibles, il a fallu consulter de multiples dossiers aux Archives départementales du Finistère, auprès du Service départemental de la publicité foncière commeaux Archives municipales de Brest pour obtenir des informations permettant de le situer dans une généalogie familiale et de décrire son statut social ${ }^{1}$.

\section{I - Une notabilité introuvable ?}

a) Une qualité de notable sans rapport avec le pouvoir politique et la fortune

Louis Sparfel n'a rien d'un notable au sens classique du terme, si l'on considère que la plupart des spécialistes de la question font de l'exercice durable d'un mandat politique la pierre angulaire de la domination du notable. En effet, durant toute sa carrière de dirigeant sportif, il n'a jamais reçu un seul mandat de ce genre. Et pour cause, il ne s'est présenté à aucune élection municipale entre 1953 et 1971. Cela ne tient pas seulement au fait qu'il est privé de la principale source de légitimité politique dans les années d'après-guerre : l'appartenance à un réseau de résistance qu'a mis à profit un homme comme Gaston Deferre pour asseoir son statut de notable à Marseille (Ollivier, 2015). C'est surtout lié au risque qu'il aurait couru en briguant un mandat politique : celui que sa condamnation pour collaboration économique avec l'occupant soit découverte. Condamné à deux ans de prison le 25 avril 1945, il est gracié par le décret du19 décembre 1945 et libéré quelques jours plus tard². Pourtant astreint à vivre hors de sa commune de résidence habituelle, il retourne rapidement habiter dans son quartier, ce qui suscite l'indignation du voisinage et l'irritation des milieux résistants, à tel point que le sous- préfet s'alarme des troubles à l'ordre public que pourrait générer sa présence. On comprend mieux alors qu'il n'était pas raisonnable pour lui d'exposer sa personne, même 20 ans après la fin de la guerre, en prenant part aux rivalités qui accompagnent une élection municipale. Descendre dans l'arène politique aurait pu encourager ceux qui étaient au courant de son secret à exhumer ce passé encombrant et, ce faisant, à mettre en cause publiquement sa moralité.

La participation à un corps politique n'est pas le seul fondement possible de la 
notabilité. Le pouvoir économique en est un autre. Sur ce plan, Louis Sparfel jouit d'une aisance incontestable. Formé à l'école de santé de la Marine à Bordeaux, il en sort avec les diplômes lui permettant d'exercer les professions de pharmacien et d'opticien. Il s'installe à son compte comme pharmacien libéral à Brest en 1938. De son côté, sa femme commence par s'occuper dela caisse de la pharmacie puis tient, pendant un temps, un magasin d'optique et de lunetterie. Installés dans cette ville industrielle et ouvrière, ils disposent donc d'un statut socioprofessionnel privilégié, sachant qu'en 1962 l'ensemble socio-professionnel formé par les cadres et professions libérales regroupe moins de $7 \%$ de la population locale ${ }^{3}$.

Louis Sparfel est un héritier, mais il ne s'inscrit pas dans une longue lignée d'aristocrates ou de grands bourgeois. Son père exerce le métier d'agent maritime en tant que gérant de société tandis que sa mère ne déclare pas de profession, mais détient des terrains et des immeubles dans une station balnéaire située à une vingtaine de kilomètres de leur domicile. La résidence secondaire qu'ils possèdent sur le littoral leur permet d'échapper à la précarité qui frappe ceux dont l'habitation a été ravagée pendant la Seconde Guerre mondiale. Ils ont la possibilité d'y trouver refuge plusieurs années durant, tandis que les indemnités qu'ils reçoivent du ministèrede la Reconstruction leur donnent les moyens d'aider à la réinstallation de leur fils et leur bru. En fait, la fortune qu'il accumule tout au long de sa vie active tient en bonne partie aux facilitésque ses parents lui accordent pour faire face aux destructions de la guerre et aux biens immobiliers qu'ils lui transmettent. En 1963, suite à l'héritage qu'il reçoit au décès de sa mère,il possède une pharmacie et un local d'habitation de $164 \mathrm{~m}^{2}$, un grand lotissement de $5408 \mathrm{~m}^{2}$,trois immeubles en ville, une vaste propriété en bord de mer et un grand terrain à bâtir de $4573 \mathrm{~m}^{2}$ récemment acquis dans cette même station balnéaire ${ }^{4}$.

La comparaison entre ses déclarations de revenus pour l'année $1961^{5}$ et une étude réalisée par l'Insee sur les revenus de 24000 ménages en 1962 nous apprend que les gains qu'il tire del'exploitation de sa pharmacie sont exceptionnels (Ruault, 1965). En dépit du décalage d'une année entre ces deux sources, ils sont 6,5 fois supérieurs aux revenus moyens des ménages de l'échantillon national et équivalent à 9,7 fois ceux des ménages de l'échantillon breton. Il ne faut pas seulement y voir l'effet lié à sa catégorie socioprofessionnelle d'appartenance, mais aussi le résultat de sa trajectoire personnelle. Les revenus de son ménage sont franchement au-dessus de la moyenne de son groupe socioprofessionnel : ils sont 1,7 fois supérieurs à ceux des professions libérales au niveau 
national et 3,2 fois plus élevés que ceux des cadres supérieurs et professions libérales de l'échantillon installés en Bretagne. L'ampleur de ses ressources permet à sa famille de vivre grand train. À la fin des années 1950, il possède, par exemple, trois bateaux de plaisance, dont un « Requin » et un «Dragon ». En 1958, leur prix ${ }^{6}$, pour la version de base, représente respectivement plus d'un an et dix mois du salaire moyen d'un ouvrier et plus de deux ans et dix mois du même salaire de référence (Baudelot et Lebeaupin, 1979).

Pour autant, cette richesse fait-elle de lui un notable ? Rien n'est moins sûr car l'influence qu'il exerce sur la vie locale du fait de sa fortune est insignifiante. Il emploie selon les années de quatre à cinq salariés, ce qui n'est pas suffisant pour peser sur le marché local de l'emploi et, au-delà, sur les élus qui gèrent la ville. Il n'a pas d'empire immobilier dont il ferait un usage commercial et il ne détient pas non plus d'organe de presse qui, de par son poids économique et son contenu rédactionnel, pourrait être utilisé comme instrument de pression. En conséquence, s'il y a notabilité, elle ne peut avoir été acquise que par une autre voie.

b) Un statut social avantageux lié à l'appartenance à une société nautique distinguée

Si sa profession et la richesse de Louis Sparfel interviennent dans son accès à la notabilité, cela tient au fait qu'elles l'autorisent à prendre la tête d'une société nautique prestigieuse, fondée au XIX ${ }^{\mathrm{e}}$ siècle par des membres de la bourgeoisie locale, des officiers et des cadres de la marine nationale ${ }^{7}$. En juillet 1958, il en est le secrétaire général. Il a 50 ans. Tout est alors prévu pour que la SRB fonctionne comme une organisation sélecte. Ses statuts stipulent que l'admission des nouveaux membres est subordonnée à l'obtention du parrainage de deux sociétaires, puis à l'agrément accordé par le comité de direction à l'issue d'un vote secret ${ }^{8}$. En plus de la barrière économique qu'oppose encore souvent le prix d'acquisition d'un bateau aux membres des classes populaires, de telles procédures donnent aux dirigeants les moyens d'exercer un contrôle sur le recrutement des entrants et, ce faisant, de garantir l'homogénéité sociale du club. De fait, si l'on s'en tient à la composition de son comité directeur en 1965, elle attire à elle des membres de la moyenne bourgeoisie, soit des professions libérales (agents généraux d'assurances, pharmaciens, commissaire-priseur), soit des cadres supérieurs(directeur commercial, directeur de publicité, directeur d'un cinéma), et des strates supérieures de la petite bourgeoisie (opticien, représentant de commerce, représentant industriel, etc.). 
Cette clôture sur un milieu social privilégié correspond à l'image que celle-ci renvoie vers l'extérieur, si l'on en juge par les témoignages de deux maîtres d'éducation physique qui, du fait des activités d'initiation à la voile qu'ils menaient durant les années 1960, côtoyaient de loin ses adhérents : "Cela n'était pas évident d'y entrer hein. Eux se fréquentaient, mais nous,nous étions vraiment le petit peuple. Pour fréquenter ce milieu-là, il fallait déjà être dans la bourgeoisie brestoise. On nous le faisait sentir ! ${ }^{2}{ }^{2}$ Cette représentation de la SRB comme une petite élite fortunée exprimant en différentes circonstances sa supériorité sociale peut difficilement être séparée du souci de la distinction que manifestent ensemble ses membres par le soin qu'ils apportent à leur tenue vestimentaire lors de leurs apparitions en public. Les photographies publiées dans la presse locale montrent que le port du costume et de la cravate est de rigueur dans toutes les réunions présentant un caractère cérémoniel.

Dans les années 1950, la considération que l'on reçoit d'autrui dans ce milieu résulte aussi de l'obtention des récompenses consacrant son mérite de navigateur talentueux. Elle tient aux victoires remportées dans les compétitions. À partir de la fin de cette décennie, la SRB va de plus en plus orienter ses activités vers la quête de succès de ce genre, dont les retombées symboliques profitent au club dans son ensemble. La lecture de la presse locale montre qu'en 1958 les deux fils Sparfel, qui ont 18 et 21 ans, sont déjà lancés dans une carrière de coureurs qui va les amener par la suite à participer à des compétitions de niveaux national et international en dériveurs et sur quillards de sport dans les années 1960, tant en Requin, une série nationale dans laquelle ils seront sacrés à plusieurs reprises champions de France $^{10}$, qu'en Dragon, une série restée au programme des Jeux olympiques entre 1948 et 1972 et dans laquelle ils remporteront le championnat de France et le championnat d'Europe en $1962^{11}$.

Louis Sparfel parvient à la tête du club durant l'été 1958 après avoir renversé le président en place sur fond de discordes et de rivalités, poussant l'ancienne équipe dirigeante à faire sécession en créant un yacht-club spécialisé dans la course-croisière. En apparence, il cumule bien des signes grâce auxquels on reconnaît d'ordinaire un dirigeant de club de voile classiques'inscrivant dans la continuité de la tradition socialement ségrégative du yachting. Pourtant, lecontexte de l'époque ne s'y prête pas. Des changements dans le mode de fonctionnement des clubs se dessinent dès les années 1950 et s'accélèrent dans la décennie suivante. 
c) Un contexte changeant, générateur de nouveaux enjeux pour les clubs de voile

Du fait de ces changements, les clubs fermés réunissant des propriétaires fortunés qui assurent sur leurs fonds propres les conditions matérielles de leur activité, et entretiennent avecle pouvoir municipal des relations sporadiques à la faveur d'une grande fête nautique annuelle, sont en passe de devenir minoritaires. On ne fera ici qu'esquisser les grandes lignes de ces transformations. L'essentiel est de montrer en quoi elles modifient l'environnement organisationnel des sociétés nautiques et créent des conditions favorables à l'émergence d'une notabilité proprement sportive au sein même des clubs de voile.

Ces changements consistent, d'un côté, en l'abaissement des barrières économiques et pédagogiques entravant l'accès à la pratique de la voile. Ils sont redevables en partie des innovations introduites sur le marché. Dans les années 1950, l'exploitation de matériaux synthétiques dans l'architecture navale rend possible la production industrielle en série. Cela se traduit par un abaissement considérable du prix de vente, une diminution sensible du poids des bateaux et un gain appréciable en matière de maniabilité et de sécurité. Des bateaux à prix réduit comme le Vaurien, la Caravelle ou le Corsaire mettent, a priori, la pratique de la voile à la portée du plus grand nombre (Charles et Bourdeau, 2006).

Au même moment, l'État mène déjà une politique en faveur de la voile dans le cadre de la promotion des loisirs de plein air. Cette politique s'intensifie avec l'arrivée de Maurice Herzog à la tête du Haut-Commissariat à la Jeunesse et aux Sports (HCJS) en septembre 1958. Les agents de ses services ont pour mission d'assurer une diffusion massive de sa pratique en introduisant une initiation à l'activité nautique dans différents cadres organisationnels. Leurs actions s'exercent auprès des lycéens lors de la demi-journée de plein air, puis de la demi- journée de sport à partir de 1961. Elles sont complétées par des interventions destinées aux jeunes ouvriers, aux jeunes employés et aux apprentis et, plus largement, à la jeunesse dite inorganisée à partir des centres d'initiation aux sports de plein air qui fonctionnent depuis 1953(Blondel, 2009), puis au sein des bases de plein air à dater de 1964 (Heluwart, 2010).

Ces changements facilitent l'essor des écoles de voile formellement ouvertes à tous. Après le second conflit mondial, les associations aspirant à faire de la voile un loisir populaire mettent en place un mode d'organisation qui rompt doublement avec la tradition du yachting. Il repose, d'une part, sur la mise à disposition d'un matériel collectif moyennant le paiement 
d'une inscription, ce qui soustrait la pratique de la voile à la nécessité de posséder son propre bateau ou d'être personnellement lié à un propriétaire. Il se fonde, d'autre part, sur l'encadrement des néophytes par des moniteurs spécialement formés pour dispenser un apprentissage méthodique de leurs savoir-faire. Le coût modique des dériveurs commercialisés dans les années 1950 et les aides apportées par les pouvoirs publics donnent à ces associations l'opportunité d'agrandir rapidement leur flottille et, donc d'accroître leurs capacités d'accueil.

S'ajoutant aux transformations socio-économiques générales qui favorisent l'accès aux loisirs d'une plus grande partie de la population (Chantelat et Tétart, 2007), ces changements facilitent une diffusion rapide de la pratique de la voile. L'audience de la Fédération nationale de voile en est décuplée. Entre 1952 et 1964, le nombre de licenciés est multiplié par 9,6, passant de 3115 membres à 34 611. Cette croissance se poursuit à un rythme soutenu les années suivantes au point que les effectifs doublent entre 1965 et $1970^{12}$. On fait un constat semblable pour les écoles de voile. Selon la fédération nationale, le nombre de leurs élèves serait passé d'environ 3500 en 1959 à un peu plus de 20000 en $1965^{13}$.

Ces changements sont à mettre en rapport, d'un autre côté, avec l'intervention croissante des pouvoirs publics dans le secteur de la voile de plaisance. On assiste à une plus grande connexion à l'État des clubs affiliés à la Fédération nationale de voile. Cela tient à l'existence d'un système de redistribution des ressources qui concerne, pour une part, les actions d'initiation et de perfectionnement à la voile. L'État subventionne les associations qui agissent en ce sens au titre de l'équipement en bateaux, de la formation des moniteurs et, parfois même,de l'accueil à prix réduit des jeunes gens dans le cadre des bourses de plein air. Parallèlement, il obtient que les écoles coordonnent leurs activités au sein de la fédération nationale, et que leur fonctionnement soit normalisé via une procédure d'homologation. Tenant lieu d'agrément administratif, cette procédure devient un enjeu en soi pour les associations concernées, car son obtention est nécessaire pour bénéficier des subventions de l'État (Lafabrègue, 2019). Par ailleurs, celui-ci promet aux collectivités locales, entre 1961 et 1975, une aide au financement de la construction d'infrastructures sportives dans le cadre de trois lois de programme pluriannuel (Falcoz et Chifflet, 1998). Cela se traduit çà et là par l'édification d'équipements portuaires et de locaux à terre dont profitent les clubs de voile.

Ces mécanismes de redistribution sont également activés au profit d'une politique 
sportive dont le but est de faire émerger quelques champions capables de porter haut les couleurs de la France dans les compétitions internationales. Selon les considérations de l'époque officiellement consacrées par Maurice Herzog dans Essai de doctrine du sport en 1965, une nation a d'autant plus de chances de se doter d'une élite sportive concurrentielle qu'elle peut compter sur un grand réservoir de bons régatiers. Dès lors, on assiste à une orientation d'une partie de l'aide publique et des crédits fédéraux vers les organes décentralisés de la fédération. La création du statut de conseiller technique régional en 1966 permet à l'administration centrale chargée des sports de mettre à disposition des ligues régionales des fédérations, les années suivantes, des fonctionnaires spécialisés dans la formation de moniteurs bénévoles et dans l'entraînement sportif(Dietschy et Clastres, 2006).

Des moyens en quantité importante sont donc mis à la disposition des clubs affiliés à la fédération, d'autant que les collectivités locales s'insèrent dans ce système d'aides en apportant leur propre concours au développement des activités nautiques. Or, la condition pour qu'ils en bénéficient tient à la capacité de leurs dirigeants de se placer à proximité des instances de décision et de s'insérer dans les réseaux d'influence. C'est ce que fait Louis Sparfel. Au lieu dese présenter comme le défenseur de la tradition du yachting et de résister aux changements en cours, il endosse un discours résolument moderniste. Il fait la promotion de pratiques et d'idées nouvelles en reprenant à son compte le thème de la démocratisation de la voile, tout en restant fidèle aux usages nautiques consacrés. On le voit ainsi investir plusieurs segments de la pratiquedu bateau à voile. D'un côté, il œuvre en faveur du développement des écoles de voile. D'un autre côté, il se consacre à la promotion de la voile de compétition. En quête de soutien pour financer ces activités dans un espace local qui s'apparente de plus en plus à une arène interorganisationnelle (Céfaï, 2007) à mesure que l'éventail des acteurs intéressés par la voile de plaisance s'élargit, il s'engage dans des stratégies d'acquisition de pouvoir qui revêtent un caractère notabiliaire en dépit des bouleversements rapides qui affectent ce secteur.

\section{II - Une notabilité fonctionnelle}

a) Un ancrage local permettant de tisser des alliances

Si l'on peut reconnaître un caractère notabiliaire au pouvoir qu'acquiert Louis Sparfel au cours de sa longue carrière de dirigeant associatif, cela tient d'abord à son ancrage dans l'espace local, un élément qui est reconnu dans la littérature comme l'un des traits les plus 
saillants de la notabilité (Briquet, 2012 ; Bensoussan, 2015). Il parvient à tisser des relations interpersonnelles directes avec les adhérents de la SRB, qui se transforment en réseau d'alliances et de solidarités. Elles donnent forme à une coalition associative qui s'efforce d'exercer un contrôle collectif sur l'essor des activités nautiques dans l'espace local en cherchant à se rendre incontournable. Ces efforts coordonnés visent d'abord à contrôler l'accès à la direction du club lui-même. Cette action conjointe permet à Louis Sparfel d'en arracher la présidence en 1958. C'est aussi grâce à la confiance que lui accordent les autres membres qu'il peut, par le jeu des procurations, renverser en 1971 le président du club dont les initiatives lui déplaisent. On n'ira pas chercher plus loin une confirmation supplémentaire de l'ascendant qu'il exerce sur la vie du club. Il suffira d'indiquer que c'est lui qui avait pris l'initiative d'installer cette personne à la tête de la SRB quelques mois plus tôt, au moment où il voyait dans ce directeur commercial régional les qualités de gestionnaire qu'il recherchait pour le club.

Les années suivantes, la SRB constitue une tête de pont à partir de laquelle s'instaure une coalition de plus grande envergure enjambant les cloisons officielles qui séparent les associations. S'inspirant de la proposition interactionniste (Clarke, 1991), selon laquelle les frontières entre les organisations formelles sont poreuses et floues, la notion de coalition associative est employée pour rendre compte de la collusion entre acteurs occupant des positions dirigeantes dans des associations formellement distinctes. Composé d'au moins six dirigeants de la SRB, elle étend son périmètre d'influence à mesure que ses membres multiplient leurs engagements bénévoles dans les instances dirigeantes d'autres organisations,qu'il s'agisse d'une association transversale comme l'OMS ou d'associations nautiques de niveau départemental, régional, ou même national, puisque plusieurs d'entre eux sont délégués de la ligue régionale à l'assemblée générale de la fédération.

Le pouvoir qu'acquiert Louis Sparfel n'est donc pas l'aboutissement d'une aventure solitaire. Même s'il est, à certains égards, un spécialiste du cumul des mandats et qu'il se distingue par sa capacité de circuler entre six organisations différentes au cours de sa carrière de dirigeant sportif, il n'aurait pas réussi à conserver un contrôle sur la direction des associations locales dont il avait assuré la présidence auparavant s'il n'avait pas pu compter sur les alliances passées avec d'autres membres de la SRB. Ceux-ci prennent sa relève une fois qu'il abandonne la présidence de l'une des deux associations nautiques locales pour prendre une responsabilité dirigeante dans une autre organisation, ou alors ils viennent 
l'épauler dans ses nouvelles fonctions en intégrant à ses côtés le comité directeur de cette dernière.

Les soutiens qu'il reçoit de leur part ne sont pas pour autant accordés sans la fourniture de contreparties. Quand bien même les relations d'homme à homme qu'il établit avec eux ne sont jamais présentées sous la forme d'un échange donnant donnant, il ne peut s'assurer de leur fidélité sans leur garantir un certain nombre de motifs de satisfaction. Il s'agit, d'une part, des contributions opérationnelles qu'il apporte à la vie du club sous la forme, par exemple, de multiples participations aux jurys de course que permet sa formation de jury fédéral, de la mise en sécurité des concurrents sur l'eau ou d'une présence fréquente dans les comités de course, toutes choses qui rendent possible l'organisation des compétitions auxquelles sont généralement attachés les membres d'une société des régates. Il s'agit, d'autre part, de sa présence assidue aux réunions des clubs qu'il dirige. En plus de témoigner de l'intérêt pour leurs occupations, il apporte des réponses aux demandes d'information formulées par les adhérents et les tient au courant de la manière dont la municipalité compte traiter les problèmes qui affectent leur activité nautique (envasement du port, réduction des places de mouillage, aménagements prévus dans le nouveau port de plaisance...). Enfin, on ne peut passer sous silence les services et les avantages collectifs de toutes sortes (nouveaux locaux, accès à un restaurant de club, etc.) que ses interventions auprès des autorités politicoadministratives et/oudes autorités sportives procurent aux adhérents de ces deux associations. b) Deux activités d'interconnexion typiques de la notabilité locale

Compte tenu de la position hyper-périphérique qu'il occupe dans le système de décision politico-administratif, un dirigeant associatif local n'exerce pas de contrôle direct sur les décisions de financement ou de redistribution de biens collectifs, mais il peut tenter de les influencer en intercédant auprès des autorités locales. Effectivement, comme l'a noté JeanLouis Briquet (2012) à propos de la recomposition de la notabilité dans la sphère politique, le pouvoir du notable tient désormais à sa capacité de tirer parti des médiations qu'il établit entre les bureaucraties publiques et le secteur d'activité qu'il représente au niveau local. C'est précisément ce qui a fait le succès de Louis Sparfel. Celui-ci réunit peu ou prou les deux conditions retenues par Pierre Grémion (1976) pour déterminer ce qu'est un notable. Il offre des garanties suffisantes de représentativité de la société locale pour accéder aux échelons de direction des administrations et briguer plus facilement l'octroi de ressources (subventions, 
aide logistique, mise à disposition de locaux, etc.) au profit des associations locales qu'il dirige, quitte à solliciter un traitement particulier nécessitant un assouplissement de la règle.

Les réponses favorables apportées aux demandes de subventions adressées par Louis Sparfel au maire de la ville pour la création de l'école de voile de la SRB en 1959, puis celle du CFNB en 1960, pourraient en offrir une illustration. Dans les deux cas, les contacts sont établis directement avec les élus locaux en dehors du circuit de décision passant officiellement par l'OMS. Ceci se comprend si l'on sait que chacune de ces deux associations n'était pas encore enregistrée auprès de l'OMS au moment où s'est décidée la répartition des crédits entre les différents clubs sportifs qui en sont membres. Cet organisme n'a donc pas pu proposer de leur attribuer, pour leur première année de fonctionnement, une portion de la subvention qui luiest versée chaque année par la ville. Néanmoins, une consultation des décisions de financement prises par le conseil municipal au profit d'autres clubs sportifs montre que cette exception à la règle commune n'est pas si rare. Il est d'usage d'accorder dans l'urgence une subvention à un club pour l'aider à financer une dépense extraordinaire. En définitive, une aide exceptionnelle répétée dans le temps constitue un indicateur plus sûr d'un traitement de faveur. Les nombreuses subventions apportées, entre 1961 et 1968, au CFNB en réponse aux multiples demandes d'aide de son président destinées à financer ses investissements et ses déficits de fonctionnement correspondent bien davantage à ce qu'on peut appeler un privilège ${ }^{14}$.

Il faut dire que l'activité d'intercesseur que déploie Louis Sparfel est, au début tout du moins, largement favorisée par les relations de coopération qu'il noue avec les administrations locales pour les aider à faire de la commune un haut lieu de l'initiation à la voile. Celui-ci n'est donc pas toujours en position de demandeur. Il apparaît en certaines occasions comme celui qui contribue, avec d'autres, à trouver une solution au problème que rencontrent les acteurs publics en mettant à leur disposition ses propres ressources. C'est le cas lors de la création en mars 1958 du Centre d'initiation au nautisme pour laquelle la SRB accorde son aide, répondant ainsi à une sollicitation du Haut-Commissariat à la Jeunesse et aux Sports ${ }^{15}$. Ce partenariat se poursuit lors de la création du CFNB qui vise à élargir le cercle des élèves de l'école de voile aux estivants. Répondant à une suggestion du SDJS, cette démarche est approuvée par la municipalité qui a saisi tout l'intérêt d'équiper la commune d'un centre nautique au moment où au moins une quinzaine de centres de ce genre fonctionnent aux alentours. Il en va de même en novembre 1963 lorsque le CFNB apporte 
son concours au HCJS afin de mettre en place, en collaboration avec la municipalité, une expérience d'initiation à la voile au profit des apprentis, des jeunes travailleurs et des élèves du lycée technique de garçons ${ }^{16}$. On voit que le pouvoir du notable sportif ne se fonde pas seulement sur son aptitude à obtenir des bureaucraties publiques des biens et des services collectifs au bénéfice de ceux qui décident de son élection. Il tient aussi à sa capacité de mobiliser des ressources qui rendent possible la réalisation des projets qu'ont ses partenaires publics en matière de nautisme. Il n'est donc pas étonnant que les autorités se soient montrées par la suite sensibles aux sollicitations de Louis Sparfel.

Les élus de la Ville sont d'autant plus attentifs aux demandes qu'il formule au nom du CFNB que la municipalité a imposé, dès le début, la présence de trois adjoints dans son conseil d'administration. Louis Sparfel a donc la possibilité de cogérer les affaires du CFNB avec des élus de la Ville sans avoir à multiplier les démarches en direction du bureau du maire : il peut leur présenter ses prétentions financières, faire connaître ses doléances quand survient un conflit avec le SDJS à propos de l'usage des locaux, rechercher avec eux une solution acceptable auxproblèmes financiers récurrents que rencontre ce centre nautique, etc.

c) Les avantages procurés par le cumul des mandats

À l'image de ce que Cesare Mattina (2004) a observé pour la notabilité politique, le statut de notable sportif est nettement affermi par la capacité d'un dirigeant local de se faire une place dans les instances de décision qui exercent un contrôle sur l'attribution d'avantages destinés aux associations locales. De leur côté, Crozier et Thoenig (1975) et Pierre Grémion (1976) ont bien noté que le cumul des mandats permet de se positionner favorablement dans les jeux de pouvoir qui se déroulent au sein des organisations et entre organisations. Cela donne la possibilité d'accéder plus facilement à des informations, et d'exercer une influence directe sur les circuits de décision sans avoir à s'en remettre à un réseau de relations dont l'efficacité est toujours sujette à caution. Effectivement, les capacités d'interconnexion bureaucratique de Louis Sparfel sont démultipliées à partir du moment où, en plus d'être présent dans les comités directeurs de deux associations nautiques locales, il occupe un poste électif à l'OMS dont il devient vice-président à partir de 1964, et à la Ligue régionale de voile dont il est élu présidentà la fin de l'année 1966 après en avoir été vice-président pendant plusieurs années.

Le fait de siéger au bureau de l'OMS est un réel atout. D'un côté, il y côtoie des 
fonctionnaires et des élus avec qui il peut discuter des projets nautiques de la ville. D’un autre, il participe aux délibérations de l'Office. Ceci dit, la lecture des procès-verbaux des réunions montre qu'il ne bénéfice d'aucun traitement prioritaire de la part de la municipalité sur la question des équipements prévus au port de plaisance. En revanche, il parvient à peser sur le fonctionnement de l'OMS. Les inscriptions portées sur un document de travail de l'Office attestent d'une intervention permettant aux deux clubs de voile dont il est membre d'échapper en 1967 à une modification des règles de financement qui leur aurait été très défavorable. Non seulement leur recul dans l'ordre des clubs subventionnables est annulé, mais leur rival, le yacht-club, est relégué les années suivantes dans le groupe des clubs les moins bien traités ${ }^{17}$.

Ses capacités d'intervention ne seront jamais aussi importantes que pendant les six années durant lesquelles il restera à la tête de la ligue régionale de voile. Il a, alors, la possibilité de participer à de multiples jeux de pouvoir du fait de la position privilégiée qu'il occupe à l'interface entre le local et le national. Depuis cette position, il recourt à un large éventail de stratégies dans les relations qu'il noue avec les autres acteurs du monde de la voile de plaisance de façon à fournir des avantages aux associations locales auxquelles il est lié et à en priver ses rivaux.

En tant que président de la ligue régionale, il incarne l'autorité légitime que la fédération nationale a déléguée à cette instance décentralisée sur cet échelon territorial. Il est donc bien placé pour plaider la cause de la SRB lorsqu'il est question de répartir entre les différents clubs de la ligue l'organisation des épreuves régionales. On ne s'étonnera donc pas qu'au cours de cette période la SRB reçoive la mission d'organiser plusieurs championnats de ligue sur les supports où certains de ses adhérents se montrent à leur avantage.

Cependant, les opportunités de gain qu'offre la direction de la ligue ne se limitent pas au niveau régional. L'accès à cette fonction insère son titulaire dans des réseaux de relations constitués sur des bases formelles ou coutumières, qui raccordent ce dernier aux plus hautes instances de l'appareil fédéral. Il peut, ainsi, peser sur les décisions qui y sont prises sachant que les présidents de ligue rencontrent le président de la fédération quelques fois par an et qu'il est d'usage de les laisser assister au comité directeur aux côtés de ses membres officiels. Ceci explique sans doute qu'il peut servir aussi bien les intérêts des associations locales à la direction desquelles il est associé. Cette action d'influence s'opère selon deux modalités. 
D'un côté, cette position accroît sa capacité de réduire la gamme des cours d'action ouverts aux clubs locaux qui sont susceptibles de faire de l'ombre à la SRB (Chazel, 1974). En effet, elle lui permet de nuire aux intérêts du groupe des régatiers qui font sécession en 1967 pour fonder leur propre club. Localement, les dirigeants de la SRB font le nécessaire pour les priver de la possibilité de se doter d'un plan d'eau officiel, une condition requise pour qu'un club puisse être affilié à la fédération et que ses adhérents disposent d'une licence fédérale, indispensable pour participer aux régates officielles. En outre, sa présence au comité directeur de la fédération permet à Louis Sparfel d'intriguer pour retarder le plus possible l'octroi de cette affiliation qui intervient, finalement, 18 mois après la déclaration de ce club à la préfecture ${ }^{18}$.

D'un autre côté, il réussit, de manière directe ou indirecte, à faire profiter la SRB de multiples bénéfices. Dès le début de sa mandature, il obtient que l'un des cinq centres fédéraux de perfectionnement à la régate que la fédération a décidé d'ouvrir dans le pays soit installé à Brest et qu'il soit doté d'un conseiller technique régional ${ }^{19}$. En outre, il réussit à convaincre les autorités sportives de confier à la SRB l'organisation de deux championnats de niveau international, ce qui renforce, par contrecoup, son prestige.

Par ailleurs, le fait de présider la ligue régionale lui confère une plus grande légitimité auprès des édiles locaux. Du fait de l'accroissement de sa surface de représentativité, les démarches qu'il effectue auprès du maire retiennent toute l'attention de ce dernier, ce qui se traduit par le soutien important que la collectivité locale accorde à la SRB à cette occasion (versement de subventions, mise à disposition de moyens matériels, intervention auprès des autorités militaires pour bénéficier du concours de la Marine nationale, etc.).

Pour autant, le statut de notable ne se réduit pas à la mise en œuvre de stratégies gagnantes de partenariat permettant à un dirigeant sportif d'aiguiller des avantages vers les associations qu'il administre. Pour y parvenir, celui-ci doit faire la preuve de sa crédibilité.

\section{d) Assurer sa crédibilité}

La crédibilité du notable sportif se repère à son aptitude à servir l'intérêt général dès lors que la pratique de la voile de plaisance est en passe d'être reconnue comme un bien public d'intérêt local. S'il veut emporter l'adhésion de ses interlocuteurs, il doit donc savoir faire des déclarations et des actions symboliques qui soient perçues comme cohérentes et pertinentes avec la manière dont est définie la situation du nautisme en tant que problème 
public. Cela suppose de sa part de disposer des compétences rhétoriques et dramaturgiques nécessaires pour convaincre les instances politiques et administratives qui exercent leurs prérogatives dans l'espace local de l'aptitude des organisations associatives qu'il représente à concourir au bien public. Il doit donc composer avec les contraintes de publicisation en puisant dans un répertoirede justifications typiques et dans une sorte de grammaire d'usage, de façon à rendre compréhensibles et normativement acceptables ses façons de parler et d’agir (Céfaï, 2002).

Une analyse des démarches faites par Louis Sparfel en 1959 et 1960 auprès de la municipalité pour obtenir des subventions au profit des écoles de voile de la SRB, puis du CFNB montre que le succès de ses entreprises est lié à sa maîtrise des justifications établies de longue date dans le secteur du sport et du plein air. Elles ont la vertu de faire sens pour ses interlocuteurs et d'être jugées suffisamment valides, sur un plan normatif, pour autoriser un acteur public à se joindre à une initiative privée, puisqu'il s'agit, en fin de compte, de fabriquer ensemble du bien public à l'échelle locale. L'argumentation employée pour les convaincre que son projet est digne de leur soutien ne fait qu'appliquer localement et étendre à un secteur d'activité particulier la voile de plaisance, un discours historiquement convenu.

Le premier argument présenté consiste à inscrire ces initiatives dans un effort de démocratisation faisant écho aux politiques publiques du même nom qui se multiplient à cette époque : on en trouve dans la culture (Caune, 1999) et dans le sport en général, à travers l'éducation physique (Attali et Saint-Martin, 2012). Il s'agit, ici, de faire en sorte que la voile de plaisance ne soit plus un privilège réservé aux gens riches en ouvrant l'accès des écoles de voile à tous les jeunes, toutes classes sociales confondues. Les motifs associés à cet argument central portent sur les effets bénéfiques que pourrait avoir sur la collectivité une pratique massive de la voile de plaisance. L'un renvoie aux qualités éducatives qui sont reconnues à cette activité. Celle-ci est censée contribuer au développement physique et moral de ses adeptes : «La pratique de la voile est un véritable sport, formateur pour le corps et encore plus pour le caractère. Mettre ce moyen de formation et de distraction à la portée des plus jeunes est la meilleure façon de prévenir ces manifestations d'agressivité qui inquiètent tant actuellement. Pratiquer la voile c'est posséder, pour toute la vie, un moyen d'évasion et une possibilité de détente incomparable, facteurs d'équilibre dans un monde dominé par la machine et la vitesse ${ }^{20} . »$ 
Une même rhétorique est activée dans d'autres courriers et dans les déclarations faites à la presse locale. Elle s'adosse à un discours commun sur les bienfaits de l'encadrement de la jeunesse par le biais du sport et des activités de plein air. Puisant ses racines dans les luttes qui opposent dès la fin du XIX ${ }^{\mathrm{e}}$ siècle les grands réseaux d'action socio-politique (Arnaud et Camy, 1986), celui-ci connaît un regain doctrinal sous IV République (Robène, 2018). Balançant entre hygiénisme et moralisation des mœurs, il est repris par les acteurs parlant au nom des pouvoirs publics ou travaillant avec eux, tel Maurice Herzog dans la conférence de presse qu'il tient le 11 juin 1958 pour annoncer la création du Comité national de plein air ${ }^{21}$. Le dernier motif allégué pour persuader les élus de la commune de financer les écoles de voile concerne la contribution que l'attractivité de la ville sur le plan nautique pourrait apporter à l'économie locale. Présente ailleurs dès la période d'après-guerre, dans une station comme Deauville par exemple (Dutheil et al., 2018), cette préoccupation est vive dans une région où les responsables politiques s'inquiètent de voir la jeunesse partir chercher du travail loin de là ${ }^{22}$. L'aménagement touristique de la région leur semble être une bonne solution pour fournir à la population locale des emplois permettant à la jeunesse de rester vivre au pays. L'accueil favorable réservé aux demandes de Louis Sparfel montre que les arguments qu'il présente au maire de la ville et à son adjoint aux sports sont jugés recevables.

Il est d'autres situations où la crédibilité des dirigeants sportifs et, partant, leur statut de notable, peut être avantageusement mise en scène. Il s'agit de toutes les cérémonies auxquelles donnent lieu les rassemblements liés à la vie sportive des clubs ou à celle de la ville : assemblée générale, ouverture d'une compétition, inauguration d'un équipement, visite d'un responsable politique national... Qu'ils y soient conviés en tant qu'invités de marque ou qu'ils y tiennent le rôle de principal officiant, les dirigeants sportifs ont l'occasion de paraître en public aux côtés de ceux qui occupent de hautes fonctions dans la sphère politique, dans les administrations publiques, dans les organisations sportives nationales, voire internationales, dans le secteur du tourisme ou dans les organismes consulaires.

Ces cérémonies donnent lieu à des performances dramaturgiques au cours desquelles les acteurs s'efforcent de représenter et de célébrer la cohésion de la communauté locale et la concorde entre les élus de la commune et les citoyens de bonne volonté réunis autour de la production d'un bien public. Simultanément, elles signifient officiellement à tous l'approbation de l'action conduite par les clubs qui sont honorés en ces occasions et valent donc ratification de leur capacité d'agir dans le sens de l'intérêt général d'ordre local. Ce 
faisant, elles donnent une grande visibilité à leurs réalisations et accroissent leur prestige. Ces échanges de bons procédés entrent dans «un jeu de légitimités réciproques apte à renforcer les positions de chacun des groupes d'acteurs » (Palard, 1981 : 320). Effectivement, si elles constituent un vecteur de légitimation du pouvoir local et de ses mandataires (Mandret-Degeilh, 2010), elles renforcent aussi la représentativité des dirigeants sportifs. Elles leur apportent une caution supplémentaire et théâtralisent leur respectabilité.

À force de se répéter, ces cérémonies confèrent au dirigeant sportif un statut de notable ;du moins, cette proximité avec les représentants des autorités politico-administratives sous le regard de la presse peut-elle être suffisante pour convaincre l'assistance et amener le dirigeant lui-même à croire qu'il fait partie des notables locaux. Ceci ne tient pas seulement à leur coprésence dans un cadre solennel. L'effet symbolique de cooptation que produisent ces rituels réside aussi dans leur agencement matériel qui rend manifeste la séparation entre les officiants auxquels revient le privilège de prendre la parole en public et le reste de l'assemblée auquel est assignée la place de simples participants qui regardent, écoutent et applaudissent les acteurs de la cérémonie (Cuisenier, 2006). Toutefois, ceux-ci ne sont pas que des spectateurs, ils jouent aussi le rôle de témoin de cette notabilisation et pourront l'attester ultérieurement. Cette cooptation prend une allure encore plus objective au moment de la remise d'une décoration, telle la médaille de la jeunesse et des sports reçue par Louis Sparfel en $1964^{23}$. Le contenu des allocutions qui jalonnent ces cérémonies contribue aussi à cet effet symbolique : ce sont des actes conventionnels au cours desquels les intervenants s'échangent des remerciements et des félicitations, énoncent leurs souhaits et exposent des projets, en prenant soin de rapporter leurs propos à l'intention de servir l'intérêt de la ville et de ses habitants.

Ces stratégies d'accréditation se révèlent payantes car, très vite, Louis Sparfel et les élus municipaux alignent mutuellement leurs lignes d'action autour de l'un ou l'autre de ces trois thèmes : l'accessibilité de la voile pour tous les habitants, quelles que soient leurs conditions, le développement d'une initiation à la voile, l'attractivité de la ville par le biais des activités et des équipements nautiques. Toutefois, l'efficacité symbolique de ses performances dramaturgiques et rhétoriques n'est pas suffisamment grande pour le préserver des faux-pas commis par ailleurs. C'est justement ce qui survient au début des années 1970, au moment où il semble être à l'apogée de sa carrière notabiliaire. En vérité, il s'agit d'un 
triomphe en trompe-l'œil car sa perte d'influence a débuté depuis plusieurs années.

\section{III - La perte de la notabilité}

Le statut de notable sportif n'est pas un état permanent. S'il se construit dans le temps, au travers d'une activité de dirigeant de club qui peut se prévaloir de sa représentativité pour tisser des relations privilégiées avec diverses autorités, il peut également se perdre au cours de la même activité. Dans le cas de Louis Sparfel, cette perte d'influence suit une chronologie différente suivant qu'elle affecte l'un ou l'autre club, le CFNB ou la SRB.

a) La perte de centralité du Centre de formation nautique brestois

Le notable sportif tire son pouvoir de la confiance que lui accordent les autorités dont il sollicite l'appui. Ce faisant, ces dernières se mettent en situation de vulnérabilité à l'égard de leur partenaire dont elles ne peuvent contrôler l'action. En effet, il est toujours possible que celui-ci nuise à leurs intérêts en ne répondant pas à leurs attentes (Lorenz, 2001). Dans le cas de Louis Sparfel, les premières initiatives qu'il a prises au début des années 1960 autour des écoles de voile ont certainement consolidé la confiance que les autorités politiques et administratives avaient placée en lui. Cependant, celle-ci s'inscrit dans un processus au cours duquel les partenaires apprennent l'un de l'autre jusqu'où peut aller leur coopération. Si la confiance peut se fortifier à la faveur d'une coopération réussie, elle peut aussi se dégrader quand les liens que les partenaires ont tissés suscitent le mécontentement de l'une des parties.

L'affaiblissement de la coalition constituée autour de sa personne se produit à la suite d'incidents qui mettent à mal la relation de confiance entre les dirigeants du CFNB, la municipalité et le SDJS. Il faut d'abord y voir l'effet des difficultés économiques du club, dont les élus ne cessent de solliciter l'aide de la collectivité locale pour leur permettre de régler toutes sortes de dépenses. Au-delà des demandes de subventions classiques, son président s'adresse à plusieurs reprises au maire pour bénéficier de subsides qui lui permettraient de régler les premières annuités de l'emprunt contracté pour lancer l'école de voile, ou de $s$ 'acquitter de ses frais de fonctionnement ${ }^{24}$. En définitive, cette situation montre les limites de l'exercice du pouvoir notabiliaire. À force de réclamer un traitement de faveur pour le CFNB, Louis Sparfel et ses alliés érodent l'un des ressorts de la confiance, la présomption de compétence (Perrin, 2013). Cette succession de requêtes exceptionnelles les fait apparaître, à la longue, comme des gestionnaires peu fiables. Elle conduit l'exécutif local 
à réviser leur partenariat : au bout de six ans, il procède à la municipalisation des bâtiments et de la flottille du centre ${ }^{25}$.

Cette baisse de confiance résulte aussi de la brouille avec le SDJS qui débute en 1964 par une querelle au sujet de l'accès aux locaux, alors même que le CFNB a bénéficié à plusieurs reprises de subventions de la part de cet organisme. On peut parier que cette décision unilatérale est interprétée par l'autre partie comme un manquement à une autre forme de confiance, celle fondée sur la bienveillance (Perrin, 2013) ${ }^{26}$. À la tête du CFNB, Louis Sparfel ne juge pas bon de s'impliquer volontairement pour conforter la qualité de la relation qui s'est nouée avec cette administration, en s'engageant au-delà de ses obligations formelles. Ce refus de prendre en compte les intérêts de son partenaire ne peut que nuire à sa réputation et à celle du centre nautique qu'il représente. Cet incident fâcheux se solde en 1969 par l'opposition du service départemental à la proposition qui lui est faite de coopérer avec le CFNB pour mettre en place une expérience d'initiation sur Optimist destinée aux jeunes enfants $^{27}$.

Il en résulte un affaiblissement de l'autorité morale de Louis Sparfel. L'érosion de sa crédibilité aurait pu ne pas avoir de conséquences fâcheuses si le CFNB avait conservé la place centrale qui lui avait été attribuée par la municipalité lors de sa création. Il en va tout autrement. Des changements se font rapidement sentir au niveau local : le nombre d'écoles de voile crôt fortement. Le dépouillement des Archives municipales et de la presse locale permet d'estimer à neuf le nombre d'associations nautiques disposant d'une base et/ou de bateaux d'initiation dans l'agglomération brestoise au début des années 1970, en plus de la SRB et du CFNB. Concomitamment, on observe une hausse du nombre d'organismes désireux de trouver des équipements au port de plaisance pour y faire de la voile. La prétention qu'avait Louis Sparfel, dix ans auparavant, de monopoliser la représentativité de la voile d'initiation en se plaçant à la tête du CFNB n'a donc plus de fondement, d'autant que l'exécutif local a bien saisi que ce centre ne remplit pas la mission qui lui avait été confiée de répondre aux multiples demandes émanant de la diversité des groupes composant la société locale.

Dès 1969, la municipalité projette de bâtir un centre nautique polyvalent qui pourrait accueillir des séances d'initiation et de perfectionnement, des entraînements et de grands championnats. La table ronde organisée par l'OMS en 1972 à la demande de l'adjoint aux 
sports en vue de réfléchir à la création d'un centre de ce type ne fait qu'acter les faiblesses du $\mathrm{CFNB}^{28}$.Le rapport établi en amont juge que sa fréquentation est très inférieure aux ambitions de la municipalité et fait état de l'évitement dont est l'objet le CFNB de la part des clubs d'entreprise locaux. L'officialisation de ces constats justifie que la municipalité retire sa confiance à son équipe dirigeante pour réaliser un tel projet. Dès lors, l'influence que Louis Sparfel et ses alliés peuvent espérer exercer, par le biais du CFNB, sur les décisions portant sur l'organisation des activités nautiques dans l'espace local est réduite à peu de chose.

b) L'effondrement de la Société des régates de Brest

Au début des années 1970, la SRB conserve un prestige intact. Elle continue d'être sollicitée par les autorités sportives pour monter des manifestations sportives d'envergure nationale et internationale. De leur côté, les autorités politiques, administratives et militaires persistent à honorer de leur présence les organisateurs et les concurrents lors des cérémonies d'ouverture et de remise des prix. Néanmoins, sa représentativité de la voile sportive est affectée, depuis déjà quelques années, par l'irruption de deux nouveaux clubs dans son secteur. En se manifestant dans l'espace local par l'élargissement de l'éventail des clubs avec lesquels l'exécutif local peut contractualiser, le contexte national marqué par la dissémination de la pratique de la voile réduit la dépendance de la municipalité vis-à-vis des dirigeants de la SRB pour organiser sur place de grands championnats. Celle-ci a désormais les moyens de mener à l'égard de son partenaire historique une stratégie d'autonomisation (Callède et Dané, 1991).

Concomitamment, les capacités d'interconnexion de ses dirigeants se réduisent. Leurs interventions auprès des autorités politiques et sportives n'ont pas l'efficacité souhaitée. Cela se manifeste lors de l'organisation du championnat du monde des Vauriens en 1972. Confiée au club voisin, le Club nautique de l'Elorn (CNE), cette épreuve doit se tenir, néanmoins, sur le plan d'eau qu'utilise habituellement la SRB. Les efforts que déploie Louis Sparfel, en sa qualité de président de ligue, de concert avec son fils cadet qui préside la SRB, restent vains. Ils ne réussissent pas à dissuader l'équipe municipale et, avant elle, la fédération, de soutenir ce club, puis d'obtenir que la SRB soit associée à l'organisation de ce championnat.

L'absence de connivence avec la municipalité aboutit à une crise consécutive à l'organisation par la SRB du championnat du monde des Finns en 1973. Les organisateurs 
enregistrent un déficit considérable qui dépasse de très loin la somme maximale que la municipalité a réservée au financement de cette épreuve. Avant même que la collectivité locale soit sollicitée pour prendre à sa charge la totalité des pertes de la SRB, on peut lire sur le dossier municipal consacré à cette manifestation les doutes qu'éprouvent les élus de la Ville au sujet de la capacité du comité d'organisation de mener à bien cette opération, signe que la confiance entre la municipalité et les dirigeants de la SRB est entamée ${ }^{29}$. La réaction des élus et des fonctionnaires de la Ville, une fois que les comptes de la manifestation leur sont présentés, fournit une autre indication des dispositions dans lesquelles ils se trouvent à l'égard de l'équipe de la SRB. L'inquiétude laisse la place à l'étonnement et au malaise, à tel point que le secrétaire général de la mairie se demande s'il est judicieux pour la municipalité d'honorer ses engagements en atteignant le montant maximal inscrit au budget. Si l'exécutif local accepte de faire un dernier versement, en revanche, il ne fait aucune exception pour la SRB. Celle-ci est traitée comme les autres clubs nautiques qui ont présenté des demandes similaires ${ }^{30}$. Aucune subvention supplémentaire ne lui est accordée. L'ampleur des dépenses qu'il lui est demandé de prendre à sa charge sape les dernières bases sur lesquelles reposait la confiance de la municipalité, à telle enseigne qu'elle ne manifeste aucune bienveillance à l'égard des intérêts du club en refusant, à son tour, de s'engager au-delà de ses obligations formelles.

Malgré la dégradation des relations entre les dirigeants de la SRB et la municipalité, ce déficit aurait peut-être pu être comblé si les premiers avaient eu la possibilité de trouver un soutien financier auprès d'autres partenaires. Or, le réseau de relations de Louis Sparfel établi sur des bases formelles s'est beaucoup appauvri. En 1973, il ne dispose plus de mandats dans les organes représentatifs de la voile au niveau du Département et de la Région. Cela explique sans doute qu'il méconnaisse le système de redistribution des crédits du conseil général à destination des clubs de voile, lequel passe par l'Union pour le développement des activités nautiques en Finistère (UDNF). S'il avait conservé un pied dans les instances décisionnelles de cette organisation, il aurait su que ces crédits étaient réservés aux écoles de voile et il en aurait déduit que sa demande n'avait aucune chance d'aboutir à moins de disposer d'un allié puissant en son sein. Et il aurait fallu que ce soutien soit particulièrement appuyé pour compenser la défiance qu'inspire Louis Sparfel à la direction de l'UDNF, sachant qu'il vient d'incarner pendant six ans l'organe régional de la fédération avec laquelle cette Union est en mauvais termes. Or, ce n'est pas du tout le cas. La comparaison du sort réservé à la SRB avec 
les décisions prises, à la même époque, par l'assemblée départementale concernant des demandes portées par d'autres clubs au sujet de manifestations semblables permet de prendre la mesure de la perte d'influence de Louis Sparfel à ce stade de sa carrière de dirigeant sportif.

Deux observations l'attestent. La première concerne le traitement réservé aux requêtes de la Société des régates de Douarnenez (SRD) un an avant, puis un an après la demande présentée par la SRB. On relève que la première échappe à la règle appliquée à la seconde, puisqu'elle bénéficie d'une subvention de $5000 \mathrm{~F}$ pour l'organisation du championnat international des Dragons en $1972^{31}$ et d'une aide de 2000 F pour celle du championnat de France des Dragons en $1974^{32}$. Le fait que le conseiller général et maire de Crozon préside alors la SRD a sans nul doute compté dans ces décisions ${ }^{33}$. Le second fait est encore plus éclairant. Il a trait au traitement réservé à la demande formulée par le CNE pour obtenir du conseil général une participation au financement de l'organisation du championnat du monde des Vauriens en 1972.Il est frappant de constater combien ce club jouit d'un traitement enviable par rapport à la SRB alors qu'il partait avec un lourd handicap. Bien que n'étant pas agréé par le ministère ni affilié à l'UDNF, il bénéficie pourtant d'une subvention de $20000 \mathrm{~F}$ grâce aux interventions répétées du sénateur-maire et conseiller général de $\mathrm{Brest}^{34}$, qui se garde bien de s'engager personnellement en faveur de la SRB un an plus $\operatorname{tard}^{35}$. Ce recoupement confirme l'analyse que faisait Martine Reneaud (1995) pour la ville de Bordeaux : le contrôle de plusieurs niveaux d'administration territoriale par les mêmes élus rend hypothétique la possibilité d'émerger du système d'action local et de se maintenir dans la position de notable sportif sans recevoir l'aval du maire. Et comme Louis Sparfel n'est plus, à ce moment-là, élu à la ligue régionale de voile, il manque de relais pour obtenir une aide financière de la part de la fédération.

Ne pouvant faire face aux déficits liés à la déroute financière du championnat du monde et à la mauvaise gestion du restaurant du club, les dirigeants de la SRB perdent toute crédibilité auprès de leurs interlocuteurs. Ils n'ont pas d'autre choix que de quitter leurs fonctions.

\section{Conclusion}

En arrivant à la tête de la SRB et du CFNB à la fin des années 1950, Louis Sparfel occupe une position socio-économique privilégiée qui rappelle, à bien des égards, celle dont disposent les présidents d'autres sociétés nautiques de l'époque. Certes, un profil socio- 
professionnel de ce genre constituait un facteur déterminant pour accéder à la pratique de la voile et se sentir suffisamment assuré pour assumer la direction d'une société nautique dans un milieu qui était encore massivement bourgeois. Néanmoins, même à cette époque, le fait d'être un bourgeois n'est pas une condition suffisante pour accéder au statut de notable.

Dans cet article, nous avons cherché à savoir si la notabilisation par le sport était possible en l'absence d'engagement partisan en faveur des élus locaux, alors même que la littérature spécialisée attire l'attention sur l'importance de telles affiliations politiques (Augustin et Garrigou, 1985 ; Callède et Dané, 1991 ; Honta, 2010). Nous avons supposé que l'établissement de relations durables avec les autorités politico-administratives et les autorités sportives pouvaitsuffire à propulser un dirigeant sportif au rang de notable local. Les constats effectués à partir du cas particulier examiné ici nous laissent penser que dans cette configuration l'accès à la notabilité est relativement fragile. Deux points nous poussent à tirer cette conclusion.

Si les événements ont pu donner l'impression à Louis Sparfel qu'il allait s'installer durablement dans le statut de notable, cela tient, d'abord, à la conjoncture locale caractérisée par la rareté des clubs de voile installés dans la commune ou sur son pourtour. Tant que cette situation a prévalu, la municipalité est restée dépendante de la coalition associative constituée autour de sa personne pour faire avancer ses projets en matière de nautisme. Mais quand cette situation s'est défaite, l'équipe municipale a pu mener une politique plus autonome en nouant des partenariats avec des organisations alternatives. Toutefois, nous avons vu que le cumul des mandats, surtout quand il s'effectue au sein de la filière fédérale, permettait, dans une certaine mesure, de résister à un environnement organisationnel devenu pluraliste : parce qu'il l'a placé en position favorable pour obtenir des atouts au bénéfice du territoire local et l'a doté d'une plus grande surface de représentativité, il a permis à Louis Sparfel de conserver une parcelle de pouvoir notabiliaire. Cependant, il a été établi, ensuite, que cette position avantageuse ne permettait plus d'exercer une influence sur les élus municipaux une fois qu'il était devenu notoire que le soutien d'une grande partie du public faisait défaut à l'organisation que représentait ce dirigeant sportif, alors même qu'il est censé répondre à ses attentes. Et il en est allé de même lorsque l'ambition qu'a eu Louis Sparfel d'accroître le rayonnement de ses clubsl'a conduit à négliger les intérêts de ses partenaires publics. Où l'on voit qu'un dirigeant sportif dépourvu d'ancrage partisan et d'influence économique significative ne peut conserver la crédibilité dont dépend sa position de notable qu'à la 
condition de s'inscrire dans un système d'échanges généralisés bénéficiant aux principaux acteurs intervenant sur un territoire local (instances politiques, administrations, autorités consulaires, monde sportif, etc.).

Sur le plan théorique, cet article devrait montrer l'intérêt de rapporter les actions des dirigeants sportifs au modèle notabiliaire quand il s'agit d'analyser les relations qu'ils établissent avec les autorités publiques et le pouvoir qu'ils peuvent en retirer. Non seulement la littérature scientifique constitue un guide facilitant la description et le décryptage de ces relations, mais elle offre aussi le moyen d'estimer approximativement l'écart qui sépare l'activité concrète que déploient des dirigeants singuliers de l'idéal-type du notable, et ce, à différents moments de leur carrière, comme nous avons tenté de le montrer dans ces pages.

$\underline{\text { Bibliographie }}$

ARNAUD, Pierre ; CAMY, Jean (dir.). 1986. Naissance du mouvement sportif associatif français. Sociabilités et formes de pratiques sportives, Lyon, PUL.

ATTALI, Michaël ; SAINT-MARTIN, Jean. 2012. « Le sport dans l'enseignement de l'éducation physique et sportive française durant les années 1960 », Revue européenne des sciences sociales [en ligne], 50-1, mis en ligne le 15 juin 2015. http://journals.openedition.org/ress/1160

AUGUSTIN, Jean-Pierre ; GARRIGOU, Alain. 1985. Le rugby démêlé. Essai sur des associations sportives, le pouvoir et les notables, Bordeaux, Le Mascaret.

BAUDELOT, Christian ; LEBEAUPIN, Anne. 1979. «Les salaires de 1950 à 1975 », Économie et statistique, $\mathrm{n}^{\circ} 113$, p. 15-22.

BENSOUSSAN, David. 2015. «Des notables bien vivants. La pérennité de la notabilité traditionnelle en Bretagne dans l'entre-deux-guerres », Histoire@ Politique, n² 25, p. 53-64. BLONDEL, Yohan. 2009. "L'éducateur sportif dans le secteur postscolaire : une nouvelle approche historique des formations, 1943-1961 », in Denis BERNARDEAU-MOREAU et Cécile COLLINET (dir.). Les éducateurs sportifs en France depuis 1945. Question sur la professionnalisation, Rennes, PUR, p. 81-108.

BRIQUET, Jean-Louis. 2012, Notables et processus de notabilisation en France aux $X I X^{e}$ et $X X^{e}$ siècles, consultable à https://hal.archives-ouvertes.fr/hal-00918922

CALLEDE, Jean-Paul ; DANE, Michel. 1991. Sociologie des politiques sportives locales. 
Trente ans d'action sportive à Bègles, Talence, Maison des sciences de l'homme et d'Aquitaine.

CALLEDE, Jean-Paul. 1995. « Les premiers clubs sportifs à Bordeaux, la "vocation" de dirigeantet le renouvellement des élites locales », in Jean-Pierre AUGUSTIN, Jean-Paul CALLEDE (dir.), Sport, relations sociales et action collective, Talence, Maison des sciences de l'homme et d'Aquitaine, p. 529-540.

CALLEDE, Jean-Paul. 2009. «L'implication sportive dans les clubs universitaires et l'accès aux élites sociales », in Christine BOUNEAU, Caroline LE MAO (dir.), Jeunesse(s) et élites : des rapports paradoxaux en Europe de l'Ancien Régime à nos jours, Rennes, PUR, p. 173186.

CAUNE, Jean. 2006. La démocratisation culturelle, une médiation à bout de souffle, Grenoble,PUG.

CEFAÏ, Daniel. 2002. «Qu'est-ce qu'une arène publique ? Quelques pistes pour une approche pragmatiste », in Daniel CEFAÏ, Isaac JOSEPH, (dir.), L'Héritage $d u$ pragmatisme, La Tour d'Aigues, Éditions de l'Aube, p. 51-82.

CEFAÏ, Daniel. 2007. Pourquoi se mobilise-t-on ? Les théories de l'action collective, Paris, La Découverte/Mauss.

CHANTELAT, Pascal ; TETART, Philippe. 2007. «La première sportivisation », in Philippe TETART (dir.), Histoire du sport en France. De la libération à nos jours, Paris, Vuibert, p. 33- 61.

CHARLES, Daniel ; BOURDEAU, Henri. 2006. 100 ans de nautisme, Paris, Fédération des industries nautiques, Éditions PC.

CHAZEL, François. 1974. «Pouvoir, cause et force », Revue française de sociologie, vol. $15, n^{\circ} 4$, p. $441-457$.

CLARKE, Adèle. 1991. «Social Worlds and Arenas Theory as Organizational Theory », in David R. MAINES (dir.), Social Organization and Social Process: Essays in Honor of Anselm Strauss, New York, Aldine de Gruyter, p. 119-158.

CROZIER, Michel ; THOENIG, Jean-Claude. 1975. «La régulation des systèmes organisés complexes. Le cas du système de décision politico-administratif local en France », Revue française de sociologie, vol. 16, $\mathrm{n}^{\circ} 1$, p. 3-32.

CUISENIER, Jean. 2006. Penser le rituel, Paris, PUF. 
DIETSCHY, Paul ; CLASTRES, Patrick. 2006. Sport, société et culture en France du XIX ${ }^{e}$ siècle ànos jours, Paris, Hachette Livre.

DUTHEIL Frédéric ; LEMONNIER, Jean-Marc ; FORTUNE, Yohann. 2018. « Pour une remise à flot de Deauville : le sport et les loisirs balnéaires au service de la reconstruction (1946-1958) », in Frédéric DUTHEIL, Yohann FORTUNE et Jean-Marc LEMONNIER (dir.). Reconstructions physique et sportive en France sous la IV République (1946-1958), Caen, Presses universitaires de Caen, p. 161-178.

FALCOZ, Marc, CHIFFLET, Pierre. 1988. « La construction publique des équipements sportifs :aspects historique, politique et spatial », Les Annales de la recherche urbaine, $\mathrm{n}^{\circ} 79$, p. $14-21$.

GREMION, Pierre. 1976. Le pouvoir périphérique. Bureaucrates et notables dans le système politique français, Paris, Seuil.

HALEVY, Daniel. 1930. La fin des notables, Paris, Grasset.

HELUWAERT, Michel. 2010. Jeunesse \& Sports. 1936-1986 : du militant au fonctionnaire, Paris, L'Harmattan.

HONTA, Marina. 2010. Gouverner le sport, Grenoble, PUG.

LAFABREGUE, Claude. 2019. «Conflits, alliances et négociations autour de la prise en compte des écoles de voile par la Fédération nationale de voile (1945-1976) », Sciences sociales et sport, vol. 13, $\mathrm{n}^{\circ} 1$, p. 29-56.

LORENZ, Edward. 2001. « Confiance interorganisationnelle, intermédiaires et communautés de pratique », Réseaux, $\mathrm{n}^{\circ} 108$, p. 63-85.

MANDRET-DEGEILH, Antoine. 2010. «Un événement dans l'événement : la remise de récompenses sportives par les autorités politiques locales », communication au colloque $L e$ sport transformé en événement. Usages politiques et profits symboliques, Strasbourg, 14 et15 janvier.

MATTINA, Cesare. 2004. «L'intermédiation politique des présidents de comités d'intérêt de quartier : le territoire de la notabilité », in Pierre FOURNIER et Sylvie MAZZELLA (dir.), Marseille, entre ville et ports, Paris, La Découverte, p. 82-96.

MATTINA, Cesare. 2004. «Mutations des ressources clientélaires et construction des notabilités politiques à Marseille (1970-1990)», Politix, vol. 17, n 67, p. 129-155. 
MATTINA, Cesare. 2016. Clientélismes urbains. Gouvernement et hégémonie politique à Marseille, Paris, Presses de Sciences Po.

OLLIVIER, Anne-Laure. 2015. « Notabilité et modernité politique. Le cas de Gaston Defferre, 1944-1986 », Histoire@ Politique, n 25, p. 103-119.

PALARD, Jacques. 1981. « Rapports sociaux, stratégie politique et vie associative », Sociologiedu travail, $\mathrm{n}^{\circ}$ 3, p. 308-324.

PERRIN, Christelle. 2013. «La nature de la relation entre gouvernement et associations : le rôle de la confiance », RIMHE : Revue interdisciplinaire management, homme \& entreprise, $\mathrm{n}^{\circ} 6$, p. 92-105.

PHELIPPEAU, Éric. 2002. L'Invention de l'homme politique moderne. Mackau, l'Orne et la République, Paris, Belin.

RENEAUD, Martine. 1995. La vie sportive locale : des pratiques notabiliaires aux pratiques managériales ?, in Jean-Pierre AUGUSTIN, Jean-Paul CALLEDE (dir.)., Sport, relations sociales et action collective, Talence, Maison des sciences de l'homme d'Aquitaine, p. 555561.

ROBENE, Luc. 2018. « Le projet gaulliste pour la jeunesse au temps du rassemblement du peuplefrançais (RPF) : sport, éducation physique et loisirs au cœur des enjeux idéologiques et politiques (1947-1951) », in Frédéric DUTHEIL, Yohann FORTUNE, Jean-Marc LEMONNIER (dir.), Reconstructions physique et sportive en France sous la IVe République (1946-1958), Caen, Presses universitaires de Caen, p. 219-232.

ROBERT, Michel. 1977. «Capitalisme et métamorphoses du notable », Études rurales, $\mathrm{n}^{\circ}$ 65, p. 121-128.

RUAULT, Jean-Pierre. 1965. «Les revenus des ménages en 1962 », Études et conjoncture, n 12,p. 3-112.

SCHOTTE, Manuel. 2016. «Monter en première division : trajectoires de notabilisation des présidents de clubs de football professionnel (1960-1999) », Politix, n 114, p. 99-120

TUDESQ, André-Jean. 1993. « Le concept de notable et les différentes dimensions de l'étude des notables », Cahiers de la Méditerranée, n 46-47, 1993. p. 1-12. 
${ }^{1}$ Je tiens à exprimer ma gratitude aux personnels des services publics des archives concernés pour m'avoir guidé efficacement dans le dédale des documents relatifs aux transactions immobilières et aux propriétés foncières.

${ }^{2}$ Archives départementales (AD), 31 W 345. Épuration. Dossiers individuels numérotés et non numérotés.

${ }^{3}$ Recensement général de la population de 1962. Finistère. Résultats du dépouillement exhaustif. Population, ménages, logements, immeubles. Institut national de la statistique et des études économiques.

${ }^{4} \mathrm{AD}$, Registre des hypothèques 4 Q5/2619, relevé de formalités 4 Q3/319 et registre des transcriptions portant sur huit opérations s'étalant du 4 août 1937 au 12 mars 1954. Service de publicité foncière de Brest, fiches hypothécaires, relevés de formalités au nom de Louis Auguste Sparfel et Renée Sparfel née Boulais.

5 AD, 100W228. Direction départementale des services fiscaux. Déclarations de revenus. Années 1961 et antérieures.

${ }^{6}$ Merci à Olivier Le Carrer pour m'avoir transmis ces informations tirées de la revue Bateaux de l'année 1958.

${ }^{7}$ AM, 3R4. Livret des fondateurs des régates de Brest.

${ }^{8}$ AM, 3R19. Société des régates (1959-1973). Nouveaux statuts adoptés par l'assemblée générale du 19 septembre 1946 joints à la demande de subvention du 2 juin 1959.

${ }^{9}$ Témoignage recueilli le 15 février 2010. Les parents de madame étaient employés aux PTT et aux chèques postaux. Le père de monsieur était conducteur de travaux publics, sa mère ménagère au foyer.

${ }^{10}$ Le Télégramme, «Soixante journées de régates sont inscrites en 1963 au programme de la Ligne de Bretagnesud de yachting », 21 décembre 1962. Le Télégramme, «Au cours de son assemblée générale la Société des régates de Brest a fêté ses champions », 31 décembre 1965.

${ }^{11}$ Témoignage d'un ancien président de la SRB, recueilli le 17 juin 2015.

${ }^{12}$ Archives nationales (AN), 19880066/2. Assemblées générales.

${ }^{13}$ AN, 1988066/2\&3. Assemblées générales.

${ }^{14}$ AM, 4M373. Construction du club-house de la SRB (1973) et garantie d'emprunt.

${ }^{15}$ Le Télégramme, "Ouverture, à Pâques, de quatre centres d'initiation au nautisme dans le Finistère : Brest, Châteaulin, Quimper, Concarneau », 21 février 1957.

${ }^{16}$ Le Télégramme, "Une expérience d'initiation à la voile pour jeunes travailleurs et scolaires », 14 novembre 1963.

${ }^{17}$ AM, 3R26. Office municipal des sports, chemise « Subventions aux clubs sportifs », courrier du président du comité de gestion de l'Office municipal des sports à l'adjoint au maire chargé des sports, 29 décembre 1966.

${ }^{18} \mathrm{AN}, 19880066 / 12$. Comité de direction : procès-verbaux, 1968-1971.

${ }_{19}$ AM, 3R8. Manifestations nautiques (1961-1973). Courrier adressé au député-maire de Brest, 25 septembre 1967.

${ }^{20}$ AM, 3R19. Société des régates. Courrier adressé au maire de Brest, 28 octobre 1959.

${ }^{21}$ AN, 1988066/62. Activités de plein air. Comité national du plein air (1957-1959).

${ }^{22}$ Le Télégramme, «L'assemblée générale de la Société des régates », le 19 décembre 1950.

${ }^{23}$ Le Télégramme, «Au cours de son assemblée générale la Société des régates de Brest a fêté ses champions »31 décembre 1965.

${ }^{24} \mathrm{AM}, 3 \mathrm{R} 18$. CFNB : correspondance.

${ }^{25}$ AM, 3R18. CFNB : note du service Jeunesse et Sports de la mairie, 9 novembre 1967.

${ }^{26}$ Nous avons préféré le terme de bienveillance à celui de bonne volonté employé par l'auteure citée pour désigner l'intention d'une des parties de préserver les bonnes relations avec son partenaire en s'engageant davantage auprès de lui de façon à lui permettre de faire face à des difficultés imprévues.

${ }^{27} \mathrm{AM}, 3$ R6. Centre nautique. «Initiation à la voile sur "Optimist" », sans date.

${ }^{28}$ AM, 3R6. Compte-rendu de la réunion préparatoire de la table ronde de la Voile des clubs corporatifs, 4 février 1972.

${ }^{29}$ AM, 3R8, Manifestations nautiques (1961-1973), dossier des services administratifs de la ville.

${ }^{30}$ Idem.

${ }^{31} \mathrm{AD}, 1$ BA 117. Rapport supplémentaire du préfet et procès-verbal des délibérations : $1^{\text {re }}$ session ordinaire, 15 , 17 mai, 5 juin 1972.

${ }^{32}$ AD, 1 BA 131. Rapport supplémentaire du Préfet et Procès-verbal des délibérations : $2^{\mathrm{e}}$ session ordinaire, $2^{\mathrm{e}}$ partie, $7,15,16$ et 17 janvier 1975 .

${ }^{33}$ La presse l'atteste au moins entre 1972 et 1974 . Voir Le Télégramme, « Le championnat international des Dragons est entré dans le vif du sujet », 31 juillet 1972 et Le Télégramme, «Quelques remous à l'assemblée générale de la Société des régates de douarnenistes », 11 mars 1974.

${ }^{34} \mathrm{AD}, 1$ BA 117. Rapport supplémentaire du préfet et procès-verbal des délibérations : $1^{\mathrm{re}}$ session ordinaire, 15 , 17 mai, 5 juin 1972.

${ }^{35}$ AD, 1 BA 123. Rapport du préfet et procès-verbal des délibérations :1 ${ }^{\text {ère }}$ session ordinaire, 14-15 mai 1973. 